\section{Kleine Merkelzellkarzinome nicht adjuvant lokal bestrahlen?}

\author{
Ob Patienten mit kleinen Primärtumoren eines Merkelzellkarzinoms nach \\ der Exzision von einer adjuvanten lokalen Radiatio profitieren, ist unklar. \\ US-amerikanische Dermatologen haben Hinweise gefunden, wonach die \\ rein chirurgische Lokaltherapie genügt
}

$\mathrm{M}$ erkelzellkarzinome sind mit einer Inzidenz von bundesweit etwa 300 Neuerkrankungen im Jahr selten, aber potenziell hochmaligne. Die Tumoren wachsen häufig schnell und aggressiv. Die meisten Patienten haben zum Zeitpunkt der Diagnose ein klinisch auf den Primärherd beschränktes Krankheitsstadium. Die Ansichten darüber, wie der Primärtumor am besten zu behandeln ist, gehen in der Literatur allerdings auseinander. Zwar ist die weite lokale Exzision unstrittig. Doch der Nutzen einer adjuvanten lokalen Bestrahlung, um Lokalrezidive zu verhüten, wird bezweifelt - zumindest was kleine Primärtumoren betrifft.

Dermatologen aus den USA haben deshalb 104 Patienten mit 105 primären Merkelzellkarzinomen, die weniger als $2 \mathrm{~cm}$ Durchmesser hatten, in eine Beobachtungsstudie einbezogen. Die Tumoren wurden exzidiert, eine adjuvante lokale Radiatio fand nicht statt. Die Raten an lokalen Rezidiven und Satelliten- metastasen nach alleiniger chirurgischer Behandlung waren so niedrig, dass sich für die Autoren die Frage stellt, ob es der adjuvanten lokalen Bestrahlung wirklich bedarf.

Insgesamt erlitten 19 Patienten einen Rückfall. Es zeigten sich drei Fälle von InTransit-Metastasen, neun regionäre Rezidive, zwei Fernmetastasen und jeweils ein Fall einer kombinierten lokalen mit einer In-Transit-Metastase, einer Satellitenund regionären Metastase und einer InTransit- plus regionären Metastase. Zwei Patienten entwickelten sowohl eine regionäre wie eine Fernmetastase. Bei sechs Patienten traten in der Folge weitere regionäre oder Fernmetastasen auf.

Die Überlebensraten nach 48 Monaten hingen vom anfänglichen Tumorstadium ab. Im Stadium IA/B betrugen Gesamt- und krankheitsspezifisches Überleben $85,0 \%$ und $94,4 \%$, und im Stadium 3A überlebten $63,2 \%$ und 78,1\%. Für das Stadium 3B erreichten beide Quoten nach 24 Monaten nur noch $50 \%$. Patien- ten anderer Stadien waren an der Untersuchung nicht beteiligt.

Die lokale adjuvante Radiatio zielt primär auf die Verhinderung lokaler Rezidive. Ein solches zeigte sich aber nur bei einem Patienten, ein weiterer Patient entwickelte eine Satellitenmetastase. Diese zwei Fälle hätte man mit einem lokalen Bestrahlungsfeld von $5 \mathrm{~cm}$ Radius allenfalls verhindern können. Ob dies auch für die fünf In-Transit-Metastasen gilt, hängt von ihrem Abstand zum Primärtumor ab. Zumindest drei davon lagen außerhalb des $5 \mathrm{~cm}$-Umkreises um den Primärherd. Den Autoren zufolge ist daher kaum anzunehmen, dass sie sich durch eine lokale Radiatio hätten vermeiden lassen.

Fazit: Eine adjuvante lokale Radiatio bei kleinen Merkelzellkarzinomen ist womöglich nicht nötig. Eine Vergleichsgruppe von Patienten mit ähnlichen primären Merkelzellkarzinomen, die kombiniert chirurgisch und strahlentherapeutisch behandelt worden wären, hat es allerdings nicht gegeben, wie die Autoren einräumen. Sie verweisen indes darauf, allein schon die niedrigen Rückfallraten legten einen Verzicht auf eine adjuvante Strahlentherapie nahe. Dr. Robert Bublak

Frohm ML et al. Recurrence and Survival in Patients With Merkel Cell Carcinoma Undergoing Surgery Without Adjuvant Radiation Therapy to the Primary Site. JAMA Dermatol. 2016;152:1001-7

\title{
Schmerzhafte Blasen an beiden Händen
}

Eine 64-jährige Frau litt seit zwei Jahren unter schmerzhafter Blasenbildung an den Handrücken beidseits. Eine topische Behandlung mit Steroiden besserte den Zustand nicht. Hautbiopsie, Urin- und Blutuntersuchungen bestätigten schließlich die Diagnose einer Porphyria cutanea tarda.

Diese ungewöhnliche Ursache einer Blasenbildung an den Händen tritt mit einer Häufigkeit von 1:200.000 auf und wird nicht selten verkannt. Differenzialdiagnostisch kommen ein bullöses Pemphigoid, ein dyshidrotisches Ekzem mit Ausbildung eines Pompholyx und eine Kontaktallergie in Betracht. An eine Porphyria cutanea tarda sollte man denken, wenn die Blasen an lichtexponierten Hautstellen auftreten und es an befallenen Stellen nebeneinander zu Blasenbildung, Erosionen, Hyperpigmentierung und Narbenbildung kommt. Der Urin ist zumeist dunkel gefärbt. Prof. Hermann FüeßI

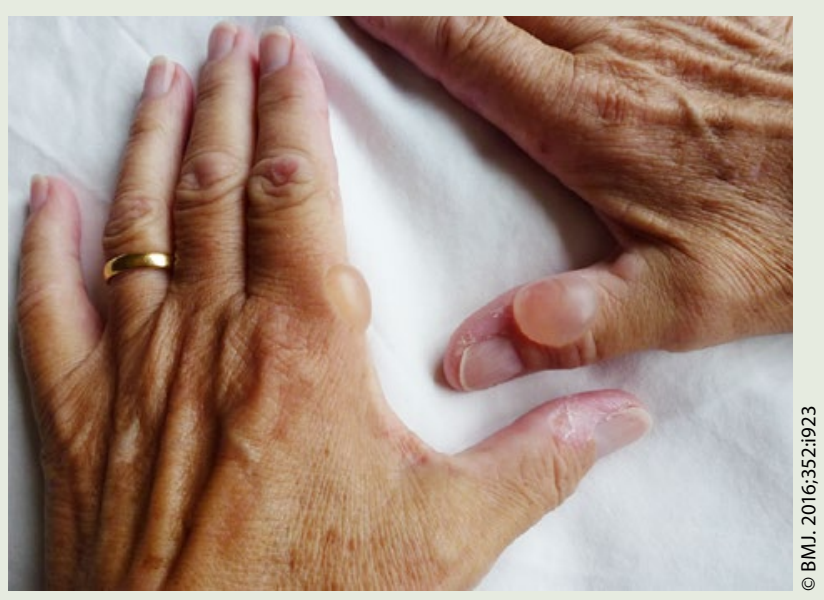

Blasenbildung an den Handrücken beidseits 